\title{
'I am excited to start my career as a qualified dentist'
}

\author{
By Priti Pankhania, 23, final year dental undergraduate, University of Leeds
}

$$
\mathrm{T}
$$
his certainly was not how I imagined my time at dental school to end. In September 2019 I was well rested from the summer break and after four challenging but enlightening years, the finish line was finally in sight. There were just a few more hurdles to jump over before I was to be rewarded with my dental qualification. Knowing that the end was within reach, I began preparing for final assessments and case presentations.

By March, I had spent many hours of both clinical and non-clinical time working on my final cases and could not wait to complete treatment plans for my patients and showcase these to my tutors in my final upcoming assessments. However, despite all of my preparations there was one challenge that I certainly had not anticipated: COVID-19. I went from treating a patient in the Restorative Clinic one afternoon to being dismissed from university life, as I knew it, overnight.

Initially, there was much uncertainty as to how my final few months at dental school would unfold. The dental school kept us informed as best as they were able to at all stages. After a few short weeks, the transition was made to online teaching and despite missing the traditional methods of learning, my experience of online teaching has been positive. Largely, I think this is because all of the online tutorials and group discussions are revision of topics that I have already been taught.

I have pondered how I would adapt to online teaching if this had happened earlier in the academic year or if I were in a younger year group, given that dentistry is very practical by nature. Though different to anticipated, I feel fortunate that we are able to adapt and progress to sit our final examinations, which we will now take online. Additionally, and reassuringly, the majority of us are still due to start our foundation training as planned in September. Although our graduation ceremony has been postponed, when it is deemed safe to do so we will still get this opportunity to celebrate our achievements and say our thank yous and goodbyes to all of the tutors who

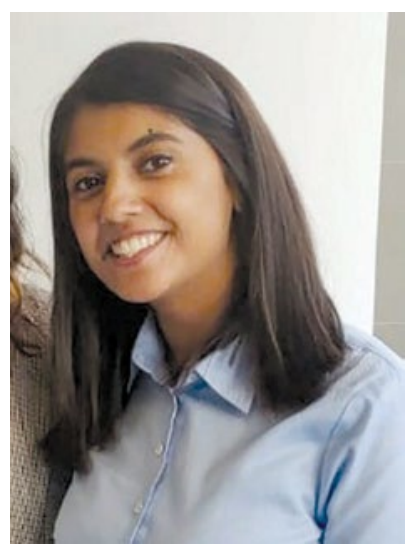

have supported us throughout undergraduate training.

Despite the unprecedented end to my undergraduate training, I am excited to start my career as a qualified dentist and to treat patients once again. In the interim period between my last clinical session as a student in March and my anticipated first clinical session as a qualified dentist in September, I am ensuring that I build a comprehensive dental knowledge base and enhance my manual and communication skills as best as I can in these challenging circumstances.

It is inevitable that there will be many changes that result from this pandemic but throughout the lockdown I have seen the profession come together in extraordinary ways to learn, grow and support one another. I firmly believe the lessons we have learned during this pandemic will make us more compassionate, resilient and ambitious dentists.

\section{Community dental officer wins BSPD award}

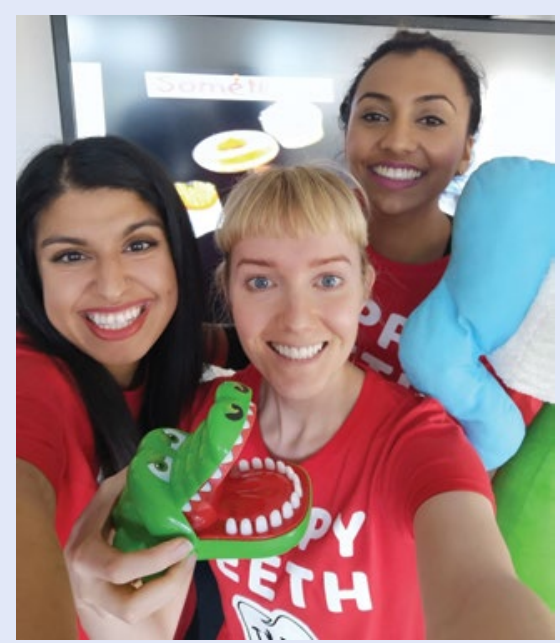

Linzi Maybin (centre) with Aruche Hamid (left) and Priya Madhas
The winner of the British Society of Paediatric Dentistry's (BSPD's) 2020 Outstanding Innovation Award is Community Dental Officer Linzi Maybin for her Happy Teeth project. Based in Yorkshire and Humber, Happy Teeth is geared to building a brighter future for children with special educational needs (SEN) or children affected by the refugee crisis.

Foundation dentists are trained to go out with Linzi to special schools to teach and screen children's teeth. Teachers and assistants in these settings are also trained in oral health so that good practice endures after the visit.

Dr Maybin said that as a student, she only had one 30 -minute lecture on the management of patients with special educational needs. After graduating, she got involved with a special school in Halifax and found the experience so rewarding she developed her Happy Teeth project. 'I just feel passionate about caring for patients who can't always communicate that they have a dental problem, said Dr Maybin.

Sarah McKaig, President of BSPD, said: 'Linzi has identified a problem and without support has created a solution. This shows compassion and drive and I am impressed by the commitment she has shown. Not only is she helping children, she is providing valuable experience to young dentists who work with her. She is inspirational'. 